\title{
Gyrostat Dynamics on a Circular Orbit: General Case of Equilibria Bifurcation and Analytical Expressions
}

\author{
Luis F.F.M. Santos, R. Melicio IEEE S. Member \\ ICT, Universidade de Évora; IDMEC, Instituto Superior \\ Técnico, Universidade de Lisboa; Departamento de \\ Física, Escola de Ciências e Tecnologia, Universidade \\ de Évora, Portugal
}

\begin{abstract}
This paper is on the study of the Dynamics of a Gyrostat Satellite on a Circular Orbit, and the achievement of the analytical expressions which describes the bifurcation of equilibria. The attitude control of modern spacecraft's is essential to fulfil its missions. Every time that is necessary to point a camera or a scientific instrument the space platform must be perfectly balanced and stabilized. When a space platform loses its ability to be balanced and stabilized, which might be due to several factors, loses also its ability to carry out its mission. One approach to achieve such conditions is using Gyrostat configuration.
\end{abstract}

Keywords-satellite; gyrostat; equilibrium; stabilization; bifurcation of equilibria

\section{INTRODUCTION}

The attitude control and stabilization of modern space platforms is crucial to the present space missions. A spacecraft or satellite requires, in particular, sensors, cameras, or lenses to perform their missions in space. Every one of those is required to point to a specific location at some random time. It is vital, that the space platforms accomplish their missions, because it is very expensive technology and of course their launching.

Like deeply developed in [1] by Santos, L., the orientation and stabilization of satellites can be made in two different ways, the first is with passive methods, which involves no power consuming, and can be achieve by taking advantage of gravity, magnetic fields, aerodynamic, rotation or even solar pressure. The second method is also called active method, which involves power consumption, and can be achieved by using fly-wheels or fuel as example. Because the passive method is power consumption free is expected that platforms which use this method have a more extended life span that the active method, but, when is required high precision and high pointing accuracy, like space-based telescopes for example, is highly recommended to use an active method in lieu of passive method. Is also expected that space platforms equipped with active methods have a lower life span, this is mainly because of a possible hardware failure or batteries run-out.

There are plenty studies made about Gyrostat equilibria and stabilization, where Sarychev V. is the main author with several relevant studies like [2-9].

\author{
A. Silva \\ Departamento de Ciências Aeroespaciais, Universidade \\ da Beira Interior, Portugal \\ ruimelicio@gmail.com
}

Nevertheless, all these studies are only for special cases, where any rotation axis coincides with orbital axis for example. For the general case only recently the first deep studies with the purpose to investigate the equilibria and stability evolution on Gyrostat platforms.

\section{MODELING}

In [2] Sarychev V. has shown that a gyrostat satellite in a central Newtonian force field in a circular orbit has no more than 24 and no less than 8 equilibria orientations. Following, the formulation of this problem starts with the consideration that a gyrostat satellite is a rigid body and has rotors which are both statically and dynamically balanced. The system representation is shown in Fig. 1.

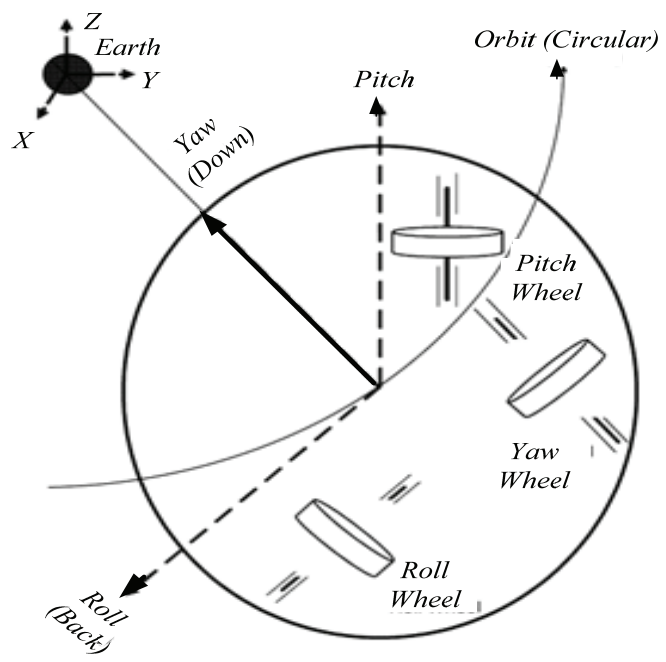

Figure 1. System representation [1].

The angular velocities of those rotors in relation to the gyrostat body are constant. Also, the center of mass of the gyrostat $O$ is the origin of a two right hand Cartesian coordinate system, being $O X_{1} X_{2} X_{3}$ the orbital coordinate system, $\mathrm{OX}_{3}$ axis is directed along the radius vector connecting the centres of mass of the Earth and of the gyrostat satellite, $O X_{1}$ axis is directed along the vector of linear velocity of the center of mass $O$. 
The gyrostat-fixed coordinate system $O x_{1} x_{2} x_{3}$, where $O x_{i}(i=1,2,3)$ are the principal central axes of inertia of the gyrostat satellite. The orientation of the $O x_{1} x_{2} x_{3}$ coordinate system with respect to the orbital coordinate system is determined by Euler angles $\psi, \vartheta, \varphi$ and the direction cosines of the axes $O x_{i}$ in the orbital coordinate system $a_{i j}=\cos \left(X_{i}, x_{j}\right)[1]$ is given by:

$$
\left\{\begin{array}{l}
a_{11}=\cos \psi \cos \varphi-\sin \psi \cos \vartheta \sin \varphi \\
a_{12}=-\cos \psi \sin \varphi-\sin \psi \cos \vartheta \cos \varphi \\
a_{13}=\sin \psi \sin \vartheta \\
a_{21}=\sin \psi \cos \varphi+\cos \psi \cos \vartheta \sin \varphi \\
a_{22}=-\sin \psi \sin \varphi+\cos \psi \cos \vartheta \cos \varphi . \\
a_{23}=-\cos \psi \sin \vartheta \\
a_{31}=\sin \vartheta \sin \varphi \\
a_{32}=\sin \vartheta \cos \varphi \\
a_{33}=\cos \vartheta
\end{array}\right.
$$

Then, the motion of the gyrostat satellite in relative to its center of mass [1] is given by:

$$
\left\{\begin{array}{l}
A \dot{p}+(C-B) q r-3 \omega_{0}^{2} a_{32} a_{33}(C-B)-\left(\bar{H}_{2} r-\bar{H}_{3}\right)=0 \\
B \dot{q}+(A-C) r p-3 \omega_{0}^{2} a_{31} a_{33}(A-C)-\left(\bar{H}_{3} p-\bar{H}_{1} r\right)=0 . \\
C \dot{r}+(B-A) p q-3 \omega_{0}^{2} a_{31} a_{32}(B-A)-\left(\bar{H}_{1} q-\bar{H}_{2} p\right)=0
\end{array} .\right.
$$

$$
\left\{\begin{array}{l}
A \dot{p}+(C-B) q r-3 \omega_{0}^{2} a_{32} a_{33}(C-B)-\left(\bar{H}_{2} r-\bar{H}_{3}\right)=0 \\
B \dot{q}+(A-C) r p-3 \omega_{0}^{2} a_{31} a_{33}(A-C)-\left(\bar{H}_{3} p-\bar{H}_{1} r\right)=0 . \\
C \dot{r}+(B-A) p q-3 \omega_{0}^{2} a_{31} a_{32}(B-A)-\left(\bar{H}_{1} q-\bar{H}_{2} p\right)=0
\end{array} .\right.
$$

In (2) and (3), $\bar{H}_{1}=\sum_{k=1}^{n} J_{k} \hat{\psi}_{k} \dot{\varphi}_{k}, \bar{H}_{2}=\sum_{k=1}^{n} J_{k} \hat{\theta}_{k} \dot{\varphi}_{k}$ and $\bar{H}_{3}=\sum_{k=1}^{n} J_{k} \hat{\varphi}_{k} \dot{\varphi}_{k} ; J_{k}$ is the axial moment of inertia of the $k^{\text {th }}$ rotor, $\alpha_{k}, \beta_{k}, \gamma_{k}$ are the constant direction cosines of the symmetry axis of the $k^{\text {th }}$ rotor in the coordinate system $O x_{1} x_{2} x_{3}, \dot{\varphi}_{k}$ is the constant angular velocity of the $k^{\text {th }}$ rotor relative to the gyrostat. Also, $A, B$ and $C$ are the principal central moments of inertia of the gyrostat, $p, q, r$ are the projections of the absolute angular velocity of the gyrostat satellite in the axes $O x_{i}, \omega_{0}$ is the angular velocity of motion of the center of mass of the gyrostat satellite along a circular orbit. Dots designate differentiation with respect to time $t$. Further on, it will be more convenient to perform a change in the nomenclature as $H_{i}=\bar{H}_{i} / \omega_{0}(i=1,2,3)$.

The next step is to ensure that the integral of energy is constant in order to proceed with the calculations.

Regarding system (2) and (3), the generalized integral of energy [1] is given by:

$$
\begin{aligned}
& \frac{1}{2}\left(A \bar{p}^{2}+B \bar{q}^{2}+C \bar{r}^{2}\right)+\frac{1}{2} \omega_{0}^{2}\left[a_{21}^{2}(B-A)+a_{23}^{2}(B-C)\right] \\
& -\omega_{0}^{2}\left(H_{1} a_{21}+H_{2} a_{22}+H_{3} a_{23}\right)+\frac{3}{2} \omega_{0}^{2}\left[a_{31}^{2}(A-C)+\right. \\
& \left.a_{32}^{2}(B-C)\right]=\text { const. }
\end{aligned}
$$

As can be verified, during the entire path of the gyrostat orbit, the several parameters remain constant, and due to that circumstance, the calculations can be preceded.

\section{EQUILIBRIUM ORIENTATIONS}

The motion of the gyrostat satellite in relative to its center of mass, setting in (2) and (3) $\psi=\psi_{0}=$ const ; $\varphi=\varphi_{0}=$ const is obtained when $A \neq B \neq C$ [1] given by:

$$
\begin{aligned}
& (C-B)\left(a_{22} a_{23}-3 a_{32} a_{33}\right)-H_{2} a_{22}+H_{3} a_{22}=0 \\
& (A-C)\left(a_{23} a_{21}-3 a_{33} a_{31}\right)-H_{3} a_{21}+H_{1} a_{23}=0 \\
& (B-A)\left(a_{21} a_{22}-3 a_{31} a_{32}\right)-H_{1} a_{22}+H_{2} a_{21}=0
\end{aligned}
$$

The system of (5) describes the equilibria of a general gyrostat satellite in a circular orbit. And, as verified, all parameters of (5) are dimensional, having 6 independent parameters. It is vital that a reduction of these parameters take place, a system like (5) is highly unmanageable and very hard to analyses.

Let's then take into consideration the following dimensionless parameters given by:

$$
v=\frac{(B-A)}{(B-C)} ; h_{i}=\frac{H_{i}}{(B-C)}
$$

Using parameters (6) into system of (5), this last one takes the following form [1] given by:

$$
\left\{\begin{array}{l}
-4\left(v a_{21} a_{31}+a_{23} a_{33}\right)+\left(h_{1} a_{31}+h_{2} a_{32}+h_{3} a_{33}\right)=0 \\
v a_{11} a_{31}+a_{13} a_{33}=0 \\
{\left[v a_{11} a_{21}+a_{13} a_{23}\right]-\left(h_{1} a_{11}+h_{2} a_{12}+h_{3} a_{13}\right)=0}
\end{array} .\right.
$$

Analyzing the system of (7), can be verified that this contains 4 dimensionless parameters, which, when compared with the system of (5) is a reduction is two parameters. This might not seem much, but this reduction has a huge impact on the calculation and computation process, it will decrease the complexity of the system of equations intensely. Exists 
now two approaches to solve the problem, the first is considering (1), system (5) and system (8), it can be derived a system of three equations with unknowns $\psi_{0} ; \vartheta_{0}$ and $\varphi_{0}$. The second and more convenient method consists in adding six conditions of orthogonality (8) for the direction cosines presented in (1) [1] is given by:

$$
\left\{\begin{array}{l}
a_{11}^{2}+a_{12}^{2}+a_{13}^{2}=1 \\
a_{21}^{2}+a_{22}^{2}+a_{23}^{2}=1 \\
a_{31}^{2}+a_{32}^{2}+a_{33}^{2}=1 \\
a_{11} a_{21}+a_{12} a_{22}+a_{13} a_{23}=0 \\
a_{11} a_{31}+a_{12} a_{32}+a_{13} a_{33}=0 \\
a_{21} a_{31}+a_{22} a_{32}+a_{23} a_{23}=0
\end{array} .\right.
$$

Following, and in order to study the equilibria, is necessary to add the system of (8) to system (7). As it was shown in Sarychev and Gutnik (1984), the second equation of (7) and the first, second, fourth, fifth and sixth equations in (8) can be solved for $a_{11} ; a_{12} ; a_{13} ; a_{21} ; a_{22} ; a_{23}$ if $A \neq B \neq C$, using dimensionless parameters (6) given by:

$$
\begin{aligned}
& a_{11}=-4 a_{32} a_{33} / F \\
& a_{12}=4(1-v) a_{33} a_{31} / F \\
& a_{13}=4 v a_{31} a_{32} / F \\
& a_{21}=4\left[v a_{32}^{2}-(1-v) a_{33}^{2}\right] a_{31} / F \\
& a_{22}=-4\left(v a_{31}^{2}+a_{33}^{2}\right) a_{32} / F \\
& a_{21}=4\left[(1-v) a_{31}^{2}+a_{32}^{2}\right] a_{33} / F
\end{aligned}
$$

where $F=h_{1} a_{31}+h_{2} a_{32}+h_{3} a_{33}$.

The determination of the direction cosines $a_{31} ; a_{32}$; $a_{33}$ allow us to achieve the remaining direction cosines through system (9). Substituting (9) in the first and third equation in (7) and adding the third equation of (8), the following three equations [1] given by:

$$
\left\{\begin{array}{l}
16\left[a_{32}^{2} a_{33}^{2}+(1-v)^{2}+v^{2} a_{31}^{2} a_{32}^{2}\right]= \\
=\left(h_{1} a_{31}+h_{2} a_{32}+h_{3} a_{33}\right)^{2}\left(a_{31}^{2}+a_{32}^{2}+a_{33}^{2}\right) \\
4 v(1-v) a_{31} a_{32} a_{33}+\left(h_{1} a_{31}+h_{2} a_{32}+h_{3} a_{33}\right) \\
\cdot\left[h_{1} a_{32} a_{33}-h_{2}(1-v) a_{31} a_{33}-h_{3} v a_{31} a_{32}\right]=0 \\
a_{31}^{2}+a_{32}^{2}+a_{33}^{2}=1
\end{array}\right.
$$

If (10) is solvable, hence (9) allows to find the other 6 direction cosines. Is important to make note that solutions of (9) exist only in the case when any two direction cosines of $a_{31} ; a_{32} ; a_{33}$ do not vanish simultaneously. Specific cases when $a_{31}=a_{32}=0 ; a_{32}=a_{33}=0 ; a_{33}=a_{31}=0$ must be examined by the direct investigation of (7) and (8), and not considering the general case.

The problem has been solved only for some particular cases when the vector of gyrostatic moment is located along the satellite's principal central axis of inertia $O x_{2}$, when $h_{1}=0 ; h_{2}=0 ; h_{3} \neq 0$ Sarychev et al. (2008), Longman (1971). Let's introduce the values $x=a_{31} / a_{33}$; $y=a_{32} / a_{33}$ and divide all terms of first equation in (10) by $a_{33}^{4}$ and second equation by $a_{33}^{3}$. Hence, the system of two equations with unknown values $(x, y)$ [1] given by:

$$
\left\{\begin{array}{l}
16\left[y^{2}+x^{2}(v-1)^{2}+v^{2} x^{2} y^{2}\right]= \\
=\left(h_{1} x+h_{2} y+h_{3}\right)^{2}\left(x^{2}+y^{2}+1\right) \\
4 v(1-v) x y+\left[h_{1} y+h_{2}(v-1) x-h_{3} v x y\right] \\
.\left(h_{1} x+h_{2} y+h_{3}\right)=0 \\
a_{31}^{2}+a_{32}^{2}+a_{33}^{2}=1
\end{array}\right.
$$

Replacing $a_{31}=x a_{33} ; a_{32}=y a_{33}$ in the last equation of the (11) is given by:

$$
a_{33}^{2}=\frac{1}{1+x^{2}+y^{2}} \text {. }
$$

Re-arranging (11) given by:

$$
\begin{aligned}
& a_{0} y^{2}+a_{1} y+a_{2}=0 \\
& b_{0} y^{4}+b_{1} y^{3}+b_{2} y^{2}+b_{3} y+b_{4}=0
\end{aligned} .
$$

Using the resultant theorem (14), the system can be presented in such matrix form [1] given by:

$$
R(x)=\left[\begin{array}{cccccc}
a_{0} & a_{1} & a_{2} & 0 & 0 & 0 \\
0 & a_{0} & a_{1} & a_{2} & 0 & 0 \\
0 & 0 & a_{0} & a_{1} & a_{2} & 0 \\
0 & 0 & 0 & a_{0} & a_{1} & a_{2} \\
b_{0} & b_{1} & b_{2} & b_{3} & b_{4} & 0 \\
0 & b_{0} & b_{1} & b_{2} & b_{3} & b_{4}
\end{array}\right] .
$$

Let's consider (14) and assuming $R(x)=0$, is obtained as 12 th order polynomial, which can be presented with the help of a symbolic matrix function in the form [1] given by: 


$$
\begin{aligned}
& p_{0} x^{12}+p_{1} x^{11}+p_{2} x^{10}+p_{3} x^{9}+p_{4} x^{8}+p_{5} x^{7}+p_{6} x^{6} \\
& +p_{7} x^{5}+p_{8} x^{4}+p_{9} x^{3}++p_{10} x^{2}+p_{11} x+p_{12}=0
\end{aligned} .
$$

In (15) when considering all polynomials becomes very huge in size, leaving no room for any possible analytical direct solution. In order to find the number of equilibria solutions and consequent equilibria positions, is necessary to follow the sequence of substituting the value of a real root of (15) into (13) then find the roots of " $y$ " in (13). For each solution of (13) one can determine two values of $a_{33}$ from (12), and then the values $a_{31}$ and $a_{32}$. Thus, each real root of (15) corresponds to two sets of values $a_{31} ; a_{32} ; a_{33}$ which, by virtue of (9), uniquely determine the remaining direction cosines $a_{11} ; a_{12} ; a_{13} ; a_{21} ; a_{22} ; a_{23}$. It follows from these considerations that the gyrostat satellite in general case $\left(h_{1} \neq 0 ; h_{2} \neq 0 ; h_{3} \neq 0\right)$ may have no more than 24 equilibrium orientations in the orbital coordinate system.

\section{BIFURCATION OF EQUILIBRIA}

Using (15), it is possible to determine numerically all equilibrium orientations of the gyrostat satellite in the orbital coordinate system and analyze their equilibria and stability. Dependence of the number of real solutions of (15), is possible to provide the numerical calculations, without breaking a generality for the case when $B>A>C$. From these inequalities it follows that $0<v<1$. The parameters $H_{1} ; H_{2} ; H_{3}$ can take on any nonzero values.

For the limiting values $v=0 \quad(A=B)$ there is an axisymmetric satellite. For this case the system of stationary equations (7) becomes simpler. And it is possible to derive from these equations the equations of circles in the plane $\left(h_{1}, h_{2}\right)$, which define the borders between the regions with the equal number of equilibria [1] given by:

$$
\begin{aligned}
& h_{1}^{2}+h_{2}^{2}=\left(4^{2 / 3}-h_{3}^{2 / 3}\right)^{3} . \\
& h_{1}^{2}+h_{2}^{2}=\left(1-h_{3}^{2 / 3}\right)^{3} .
\end{aligned}
$$

In the case of $v=1 \quad(A=C)$ there is an axisymmetric satellite. For this case it is possible to derive from (7) the equations of two astroids in the plane $\left(h_{1}, h_{2}\right)$ which define the borders between the regions with the equal number equilibria [1] given by:

$$
\begin{aligned}
& h_{2}^{2 / 3}+\left(h_{1}^{2}+h_{3}^{2}\right)^{3}=4^{2 / 3} \\
& h_{2}^{2 / 3}+\left(h_{1}^{2}+h_{3}^{2}\right)^{3}=1
\end{aligned}
$$

The coefficients of (15) depend on 4 dimensionless parameters $v ; h_{1} ; h_{2} ; h_{3}$.
The system of stationary of equation (5) depends on 6 dimensional parameters $H_{1} ; H_{2} ; H_{3} ; A ; B ; C$. For the numerical calculations decrease of the number of system parameters is very essential.

Fig. 2 shows the different equilibria bifurcation from (15). In Fig. 3 (a) and (c) are within respect to the general case, and (b) and (d) with respect to the axisymmetric case. It is important to associate both these cases; since for general case in both its extreme cases coincide with the well-known axisymmetric case, confirm that the calculations are correct.

It is interesting to trace the evolution of the obtained equilibriums as the system parameters change. From the analysis of all calculations for the mentioned above inertia parameters $v$ it follows that with increase of the $h_{3}$ values of several regions with the fixed number of equilibria become narrowed until they completely disappear. The point in the space of parameters where region with the equal number of equilibria vanishes was defined as bifurcation point. Considering the general case, the calculated bifurcation values from the different equilibria regions is shown in Fig. 3.

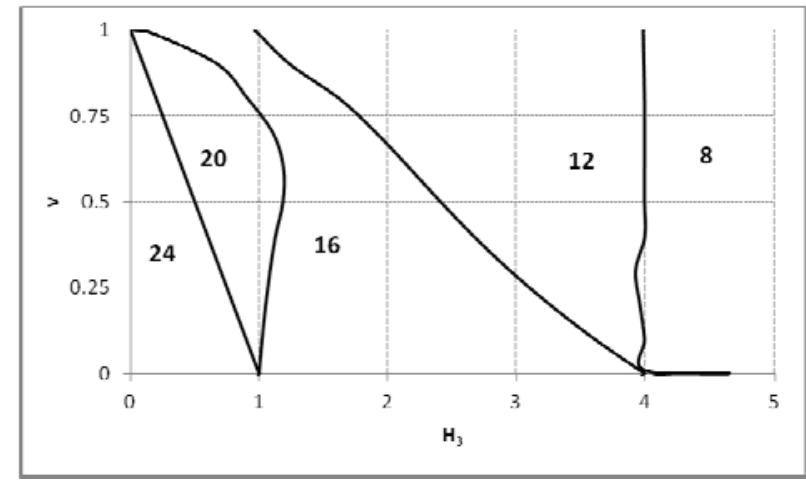

Figure 2. Bifurcation points from numerical analysis.

These results are very enthusiastic, since the complexity of the system has been broken. As can be easily seen, the $h_{3}$ bifurcation values for the regions with 24 equilibria (12 real roots) vanish in accordance with $h_{3}=1-v$. For the regions with 20 equilibria (10 real roots) the $h_{3}$ bifurcation values can be described as given by:

$$
\left\{\begin{array}{l}
v=2.913 h_{3}-2.874 ; 0.1 \leq v \leq 0.4 \\
v^{2}+h_{3}^{2}+0.802 v-0.215 h_{3}-1.794=0 ; 0.4<v<0.99
\end{array}\right.
$$

For regions with 16 equilibria (8 real roots) the bifurcation values always decrease with the decreasing of $v$ and vanish in accordance with $h_{3}=4-3 v$. The regions with 12 equilibria become smaller with the increase of values. These regions are vanishing in the center of system, coordinates for $h_{3}=4$. For $h_{3} \geq 4$ there are small regions of 12 equilibria along $h_{2}$ axes and $h_{1}$ less than $10^{-1}$. And as bigger the $h_{3}$ value, the further from the center of 

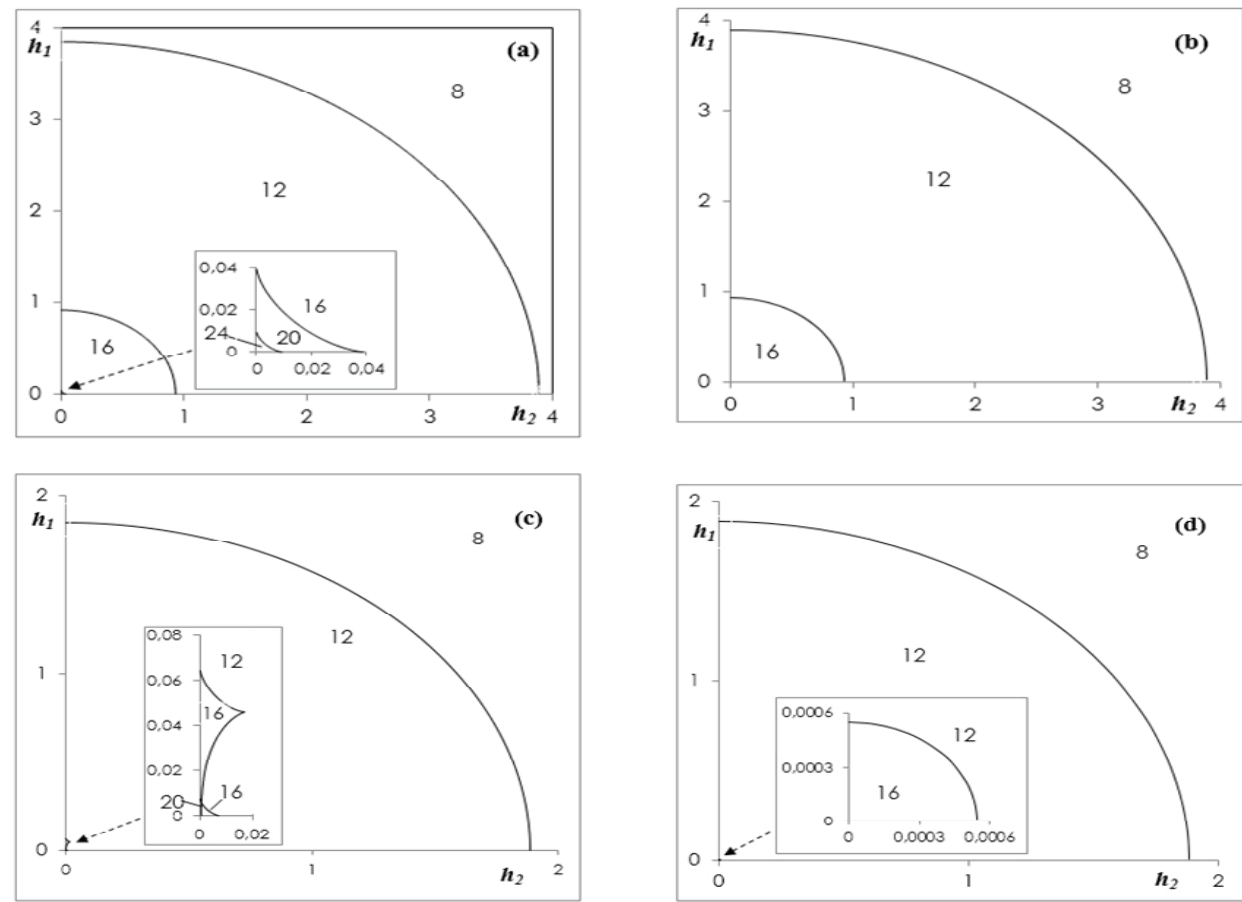

Figure 3. (a) $v=00.1, h_{3}=0.01$; (b) $v=0.0, h_{3}=0.01$ (axisymmetric case); (c) $v=0.01, h_{3}=0.99$; (d) $v=0.0, h_{3}=0.99$ (axisymmetric case).

coordinate system these small regions take position along the $h_{2}$ axis. The study of the bifurcation of equilibria of these small areas is quite important, advantages can be taken when is required to design a spacecraft with inertia parameters very close to the origin of $h_{1}$. The numerical analysis performed has permitted to achieve just that, nevertheless a dedicated paper should be addressed to that particular case.

\section{CONCLUSION}

Regarding the general case, it can be seen that whenever one principal axis is vertical and a second principal axis is perpendicular to the orbit, an Earth oriented equilibrium persists. Moreover, for a given gyrostat satellite subjected to a gravitational torque in a circular orbit, there are no more than 24 and no less than 8 equilibria positions: any of the three principal axes, each with two directions, either two transversal axes, each with two directions can point along the normal to the orbit.

This study also yields new and exciting insights relative to the bifurcation equilibria points. Numerical calculations prove that this complex gyrostat general case has a surprisingly simple bifurcation of equilibria distribution. Also, new regions of equilibria previously unknown were found, and further studies are necessary to fully understand to full potential applications of these equilibria regions.

\section{REFERENCES}

[1] L.F.F.M. Santos, Gyrostat dynamics on a circular orbit. PhD Thesis, Portugal: Universidade da Beira Interior, 2015.

[2] S.A.. Gutnik, L.F.F.M. Santos, V.A. Sarychev and A.R.R. Silva, "Equilibria of gyrostat satellite in a circular orbit," Proc. 12th Int. Conf. on Stability and Oscillations of Nonlinear Control Systems, June 2012.

[3] S.A. Gutnik, L.F.F.M. Santos, V.A. Sarychev and A.R.R. Silva, "Dynamics of a gyrostat satellite subjected to the action of gravity moment. Equilibrium attitudes and their stability," Journal of Computer and Systems Sciences International, vol. 54(3), 2015, pp. 469-482.

[4] V.A. Sarychev and S.A. Gutnik, "Relative equilibria of a gyrostat satellite," Cosmic Research, vol. 22, 1984, pp. 257-260.

[5] V.A. Sarychev and S.A. Mirer, "Relative equilibria of a gyrostat satellite with internal angular momentum along a principal axis," Acta Astronautica, vol. 49, 2001, pp. 641-644.

[6] V.A. Sarychev, S.A. Mirer and A.A. Degtyarev, "The dynamics of a satellite gyrostat with a single nonzero component of the vector of gyrostatic moment," Cosmic Research, vol. 43, 2005, pp. 268-279.

[7] V.A. Sarychev, S.A. Mirer and A.A. Degtyarev, "Dynamics of a gyrostat satellite with the vector of gyrostatic moment in the principal plane of inertia," Cosmic Research, vol. 46, 2008, pp. 61-74.

[8] V.A. Sarychev, S.A.. Gutnik, A.R.R. Silva and L.F.F.M. Santos, "Dynamics of gyrostat satellite subject to gravitational torque. Investigation of equilibria," Keldysh Institute of Applied Mathematics Russian Academy of Sciences, 2012, preprint 63:1-35.

[9] V.A. Sarychev, S.A.. Gutnik, A.R.R. Silva and L.F.F.M. Santos, "Dynamics of gyrostat satellite subject to gravitational torque. stability analysis," Keldysh Institute of Applied Mathematics Russian Academy of Sciences, 2013, preprint 25:1-36. 